\title{
Comparison of different schemes of analgosedation in patients with severe traumatic brain injury in the postoperative period
}

\author{
Y. V. Volkova, K. Y. Sharlai, K. G. Mikhnevych \\ Kharkiv National Medical University, Kharkiv, Ukraine
}

Purpose - compare different methods of analgosedation with using dexmedetomidin in patients with severe traumatic brain injury.

Materials and methods. 80 patients were diagnosed with severe traumatic brain injury. Patients in the $1^{\text {st }}$ group $(n=40)$ had analgosedation with morphine and sodium oxybutyrate, and patients in the $2^{\text {nd }}$ group $(n=40)$ were additionally treated with dexmedetomidine. The dynamics of concentration of stress markers (insulin, cortisol, glucose), HOMA index, autoantibodies to brain antigens, restoration of consciousness by the Glasgow coma scale (GCS), duration of the artificial lungs ventilation (ALV) were determined.

Results. It was found, that in the $2^{\text {nd }}$ group the reduction of the intensity of stress reactions was occured 3-4 days earlier. It was found that analgosedation with using dexmedetomidin can reduce the peak of autoantibody concentration of S-100 protein, neurospecific enolase and total cerebral antigen $(p<0.05)$. In the $2^{\text {nd }}$ group the restoration of consciousness by the GCS was faster by $24.3 \%$ the duration of ALV was less by $21.2 \%$, and a frequency of pneumonia was less by $14,3 \%$ than in patients in the $1^{\text {st }}$ group.

Conclusions. The use of dexmedetomidin may reduce an intensity of stress and autoimmune reactions in patients with severe traumatic brain injury, accelerate a restoration of consciousness by the GCS and reduce the duration of ALV.
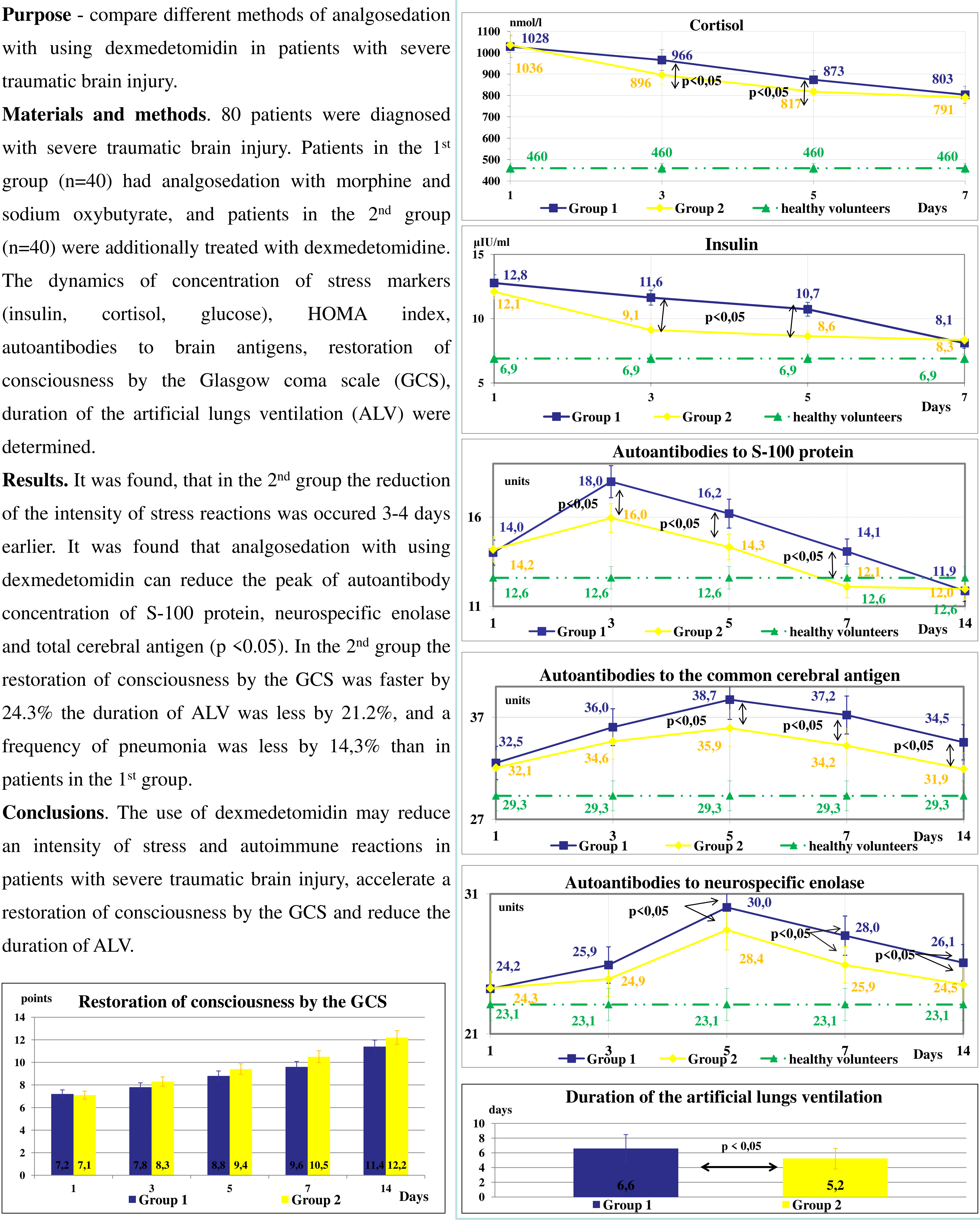